\title{
Impressum, Vol. 4, No. 6, 1977
}

S. Karger Verlag für Medizin und Naturwissenschaften GmbH, Postfach 2, 8034 Germering

Herausgeber

Prof. Dr. H. Reissigl, W. Hofrat

Vorstand des Zentralinstituts für Bluttransfusion und Immunologische

Abteilung der Universitätskliniken Innsbruck, an der Chïrurgischen

Universitätsklinik, Anichstraße 35, A-6020 Innsbruck

Prof. Dr. K. H. Bässler

Ordinarius des 2. Lehrstuhls für Physiologische Chemie an der Johannes Gutenberg-Universität Mainz, Langenbeckstraße 1, D-6500 Mainz

Prof. Dr. U. Henneberg

Geschäftsführender Direktor des Instituts für Anästhesiologie im Klinikum Steglitz der Freien Universität Berlin, Hindenburgdamm 30, D-1000 Berlin 45

Erscheinungsweise

Die Zeitschrift erscheint zweimonatlich; pro Jahr erscheint 1 Band zu

je 6 Heften.

Abonnementspreis

Bezugspreis für Jahrgang 3, 1976 DM 108,-/US\$ 49.-/SFr. 118,-. 1 Einzelheft kostet DM 24,-/US\$ 10.25/SFr. 25,-, einschließlich MwSt., zuzüglich Postgebühren.

Der Abonnementspreis ist im voraus zahlbar. Die Lieferung der Zeitschrift läuft weiter, wenn sie nicht spätestens 4 Wochen vor Abschluß eines Bandes abbestellt wird.

Sonderhefte sind nur in beschränkter Auflage vorrätig. Der Preis eines Sonderhefts ist abhängig vom Umfang. Abonnenten erhalten die Sonderhefte zu einem um 20\% vom Ladenpreis ermäßigten Vorzugs-preis.

Bereits erschienen sind

Sonderheft Nr. 1: Dextran-Symposium Krems 197360 Seiten, DM16,-

Sonderheft Nr. 2: II. Internationales Symposium, Erlangen 1973 -Bilanzierte Ernährung in der Therapie 96 Seiten, DM 20-

Sonderheft Nr. 3: Chemisch definierte Diät 36 Seiten, DM15,-Abonnements

Abonnementsbestellungen können bei jeder Buchhandlung, bei der Post oder direkt beim Verlag aufgegeben werden: Bundesrepublik Deutschland/Österreich: S. Karger GmbH, Postfach 2, D-8034 Germering/München, Tel.: (089) 844021, Telex: 524865 D, Postscheckkonto: München 40080-807 Schweiz: S. Karger AG, Arnold-Böcklin-Straße 25, CH-4011 Basel, Tel: (061) 390880, Telex 62652 CH Anzeigen

MPV med. pharm. Anzeigenverwaltung GmbH \& Co. Verlags KG, Schießstättstraße 14, Postfach 1547, D-8130 Starnberg, Telefon (08151) 13299. Gültig ist die Preisliste Nr. 3 vom 1.3. 1975.

Verlagsbeilage: Bestellkarte für die Zeitschrift (Gesamtauflage) 
Wissenschaftlicher Beirat

E. H. Abel, Stade (Verwaltung); Prof. Dr. H. Aebi, Bern (Stoff-wechsel); Prof. Dr. H. Bergmann, Linz (Anästhesie); Prof. Dr. C. Burri, Ulm (Chirurgie); Prof. Dr. H. Busch, Hamburg (Transfusions-medizin); Prof. Dr. W. Dick, Ulm (Anästhesie); Prof. Dr. P. Do-berský, Prag (Experimentelle Medizin); Prof. Dr. R. Dohrmann, Berlin (Chirurgie); Prof. Dr. S. Dudrick, Houston (Chirurgie); Prof. Dr. M. Eggstein, Tubingen (Innere Medizin); Prof. Dr. H. Egli, Bonn (Transfusionsmedizin); Prof. Dr. C. C. Ehrich, New York (Trans-fusionsmedizin); Dr. W. Fekl, Erlangen (Künstliche Ernährung); Prof. Dr. H. Förster, Frankfurt (Biochemie); Dr. P. Frank, Mainz (Pharmazie); Prof. Dr. H. E. Franz, Ulm (Nephrologie); Prof. Dr. V. Friedberg, Mainz (Gynäkologie); Prof. Dr. M. Gemperle, Genf (Anästhesie); Prof. Dr. W. Gerok, Freiburg (Innere Medizin); Prof. Dr. H. Grobecker, Bethesda/USA (Pharmakologie); Prof. Dr. A. Hässig, Bern (Hämatologie); Prof. Dr. O. Heidenreich, Aachen (Pharmakologie); Prof. Dr. A. Heidland, Würzburg (Innere Medizin); Prof. Dr. L. Heller, Frankfurt (Gynäkologie); Priv.-Doz. Dr. P.-U. Heucken-kamp, München (Innere Medizin); Prof. Dr. B. Hölscher, Berlin (Chirurgie); Prof. Dr. L. Horbach, Erlangen (Biostatistik); Prof. Dr. G. Hossli, Zurich (Anästhesie); Prof. Dr. H. Kaunitz, New York (Pathophysiologie); Priv.-Doz. Dr. K. F. Kopp, München (Nephrologie); Prof. Dr. F. Krück, Bonn (Innere Medizin); Dr. H. J. Küm-mell, Melsungen (Pharmazie); Prof. Dr. Dr. K. Lang, Bad Kro-zingen (Biochemie); Priv.-Doz. Dr. V. O. Lang, München (Pädiatrie); Prof. Dr. H. G. Lasch, Gießen (Innere Medizin); Prof. Dr. A. Lindner, Wien (Pharmakologie); Prof. Dr. P. Lundsgaard-Hansen, Bern (Experimentelle Chirurgie); Prof. Dr. H. Lutz, Mannheim (Anästhesie); Prof. Dr. H. Mehnert, München (Diabetologie); Prof. Dr. H. N. Munro, Cambridge/USA (Biochemie); Prof. Dr. J. Papenberg, Essen (Innere Medizin); Prof. Dr. K. Peter, Mannheim (Anästhesie); Prof. Dr. H. W. Pia, Gießen (Neurochirurgie); Prof. Dr. W. Rick, Düsseldorf (Klinische Chemie); Prof. Dr. G. Rodewald, Hamburg (Chirurgie); Prof. Dr. L. Róka, Gießen (Biochemie); Prof. Dr. E. Rossi, Bern (Pädiatrie); Prof. Dr. G. Schettler, Heidelberg (Innere Medizin); Prof. Dr. K. Schultis, Erlangen (Chirurgie); Prof. Dr. I. Staib, Darmstadt (Chirurgie); Prof. Dr. H. Stoeckel, Bonn (Anästhesie); Prof. Dr. W. Vahlensieck, Bonn (Urologie); Doz. Dr. E. Vinnars, Stockholm (Intensivmedizin); Dr. U. Wachtel, Hamburg (Ernährungswiss.); Prof. Dr. K. Wiemers, Freiburg (Anästhesie); Prof. Dr. A. Wretlind, Stockholm (Ernährungsphysiologie); Prof. Dr. M. Ziegler, Ludwigshafen/Rhein (Urologie); Prof. Dr. W. E. Zim-mermann, Freiburg (Chirurgie)

Schriftleitung

Hans-Dieter Viering

Waldschmidstraße 8, D-8130 Starnberg am See, Tel.: (0 8151) 6866

Lektorat: Barbara De Bernardinis, Starnberg am See

Produktion: Werner Zuckschwerdt, Starnberg am See

Satz und Druck: Buch- und Offsetdruckerei Walter Biering KG,

8 München 40

Alle Rechte, insbesondere das Recht der Vervielfältigung und Mikro-kopie sowie der Übersetzung, vorbehalten. Nachdruck, auch auszugs-weise, nur mit Genehmigung des Verlages. 